\title{
命HAD
}

ISSN-L: 2530-5115

DOI: http://doi.org/10.22585/hospdomic.v2i3.49

\section{Dos películas filmadas en el hospital Ramón y Cajal en 1978, pioneras de la nutrición artificial en España}

\section{Two films made in hospital Ramon y Cajal in 1978, seminal work in artificial nutrition in Spain}

Raimundo Garcia Garcia', José Luis Mauriz Gutiérrez²

1. Hospital Universitario San Agustín, Servicio de Urgencias, Avilés, España.

2. Universidad de León, Instituto Universitario de Biomedicina, León, España.

Correspondencia/Correspondence

José Luis Mauriz Gutierrez

Universidad de León, Instituto Universitario de

Biomedicina, León, España.

jl.mauriz@unileon.es

Recibido/Received

03.07.2018

Aceptado/Accepted

09.07.2018
Conflicto de Intereses/Competing interest No existe ningún conflictico de interés en el presente artículo.

CÓMO CITAR ESTE TRABAJO | HOW TO CITE THIS PAPER

Garcia Garcia R. Mauriz Gutierrez J L. Dos películas filmadas en el hospital Ramón y Cajal en 1978, pioneras de la nutrición artificial en España. Hosp Domic. 2018;2(3):117-27. 


\section{RESUMEN}

Se han recuperado dos películas filmadas en 16 mm en el Hospital Ramón y Cajal en 1978. Una se titula «Nutrición parenteral y enteral en un fistula yeyunal alta» y la otra «Intestino artificial (nutrición parenteral ambulante)». Son dos obras pioneras en la nutrición artificial en Ensaña. Se hacen amplios comentarios sobre la temática de las películas y su importancia en el momento actual. En las fistulas yeyunales altas la nutrición artificial, parenteral y enteral, es indicación prínceps, con eficacia comprobada. En la película «Nutrición parenteral ambulante» se muestra un modelo de nutrición parenteral ambulatoria bastante similar al actual. Se comenta que esta forma de terapia debería ser regulada por la Administración Sanitaria, hecho que hoy, cuarenta años después, todavía no ha sucedido a pesar de los cientos de pacientes con múltiples indicaciones que son sometidos a nutrición parenteral a domicilio en España cada año, con el consiguiente ahorro. Se ofrece el link para poder visualizar ambas películas.

Palabras clave: Nutrición parenteral; Nutrición enteral; Nutrición Artificial; Nutrición Parenteral Total en el Domicilio; Hospital Ramón y Cajal.

\section{ABSTRACT}

Two $16 \mathrm{~mm}$ films made in Hospital Ramon y Cajal in 1978 have been recovered. One is titled "Parenteral and enteral nutrition in a high output jejunal fistula" and the other "Artificial gut (ambulatory parenteral nutrition)". These two films are seminal work in artificial nutrition in Spain. Comments are made about the argument of both films and its importance at the present time. Artificial nutrition in high output jejunal fistulas is a main indication with demonstrated efficacy. In the film "Artificial nutrition (ambulatory parenteral nutrition)" a model quite similar to the one used at the present time is exposed. It is stated that this form of therapy should be regulated by the Spanish Medical Authority, a fact that has not happened yet forty years after although there are several hundreds of patients receiving this kind of therapy each year in Spain and it would be an important form of saving money. A link to both films is offered.

Keywords: Parenteral nutrition; Enteral nutrition; artificial nutritition; ambulatory parenteral nutrition; Hospital Ramon y Cajal. 


\section{INTRODUCCIÓN}

Se revisaron dos películas filmadas en $16 \mathrm{~mm}$, datadas en 1978, que se rodaron en el hospital Ramón y Cajal cuando este centro contaba apenas un año de antigüedad. Las películas tratan de dos temas en aquel tiempo absolutamente novedosos. Por un lado, el tratamiento de las fistulas yeyunales de alto débito, indicación princeps de la nutrición parenteral. La otra película se refiere a la nutrición parenteral ambulante. Tiene el titulo pomposo de intestino artificial. En ella se trata y se describe cómo se lleva a cabo la nutrición parenteral a domicilio, tema actualmente de mucha importancia y trascendencia. Pueden considerarse las dos películas como auténticas pioneras en nutrición artificial en España.

En 1978 empezaban a construirse hospitales y residencias sanitarias por toda la geografía española. No se había implantado aun el sistema «Médico Interno Residente (MIR)» nacional y la formación de especialistas era, cuando menos irregular. Baste como ejemplo decir que en ese año para obtener el título de especialista en cualquier materia era suficiente haberse dado de alta en el colegio de médicos en la especialidad que se quisiera durante dos años sin absolutamente ningún otro requisito ni cortapisa.

La nutrición como tal especialidad no existía en los hospitales, salvo en alguno aislado como lo era la Clínica Puerta de Hierro de Madrid donde Enrique Rojas Hidalgo se dedicaba a esta materia. En el servicio de Rojas se formó Ana Sastre Gallego, quien pasaría a formar parte de la plantilla del hospital Ramón y Cajal en 1978. Ana Sastre se dedicó a la nutrición oral y enteral pero no a la nutrición parenteral.

En Barcelona, en el hospital de Bellvitge, los miembros del Departamento de Cirugía dirigido por Antonio Sitges Creus sintieron la necesidad de aplicar nutrición parenteral en sus pacientes y dentro de su propio Departamento organizaron una Unidad de Nutricion Parenteral. En aquel Departamento de Cirugía trabajaban como adjuntos Eduardo Jaurrieta Más y Antonio Sitges Serra, quienes en aquella época organizaron algún evento académico para tratar sobre nutrición parenteral y publicaron bastantes trabajos originales sobre el tema.

En el hospital Clínico de San Carlos, en Madrid, Carlos Vara Thorbeck y Luisa García Arangüez, el primero cirujano, la segunda nefróloga, se interesaron por la nutrición artificial y escribieron un libro sobre nutrición parenteral (1). Al poco se trasladaron al Hospital Carlos Zayas de Málaga donde continuaron cultivando sus conocimientos sobre el tema.

En la costa mediterránea, en el hospital de Tarragona, Carlos Henriquez de Gaztañondo, cirujano, puso a punto algunas técnicas de colocación de catéteres intravenosos centrales para nutrición parenteral. Posteriormente se trasladó a la Residencia de la Seguridad Social de Arrecife, Lanzarote. En Pamplona, Héctor Ortiz Hurtado, cirujano de la Seguridad Social del Hospital de Navarra, estaba asimismo muy interesado en la nutrición parenteral y también Javier de Oca en la Clínica Universitaria de Navarra. Hubo otros equipos quirúrgicos por la geografía española que dedicaron sus esfuerzos a estas técnicas novedosas en mayor o menor medida.

Como puede observarse, los grupos dedicados a nutrición parenteral estaban prácticamente monopolizados por cirujanos y equipos quirúrgicos quienes, al sentir la necesidad de nutrir artificialmente a sus pacientes, cada día más graves y complejos, que no se podían alimentar por la vía oral, organizaban la preparación de las mezclas de nutrición parenteral en sus propios servicios y departamentos. En algún servicio de Cuidados Intensivos se empezó también a popularizar la nutrición parenteral, como fue en la Fundación Jiménez Diaz a cargo de Tomas Caparros y en el Hospital de Cruces de Bilbao, donde Javier Zaldumbide y el entonces médico residente Abelardo Garcia de 
Lorenzo estaban muy interesados en la nutrición parenteral. Zaldumbide, en mayo 1979, organizó la Primera Reunión de la Sociedad Española de Nutricion Parenteral y Enteral (SENPE) en Bilbao.

En el momento, actual la presencia de cirujanos en la SENPE es testimonial al haberse oficializado la administración de la nutrición artificial en los servicios y secciones de nutrición, fundamentalmente ocupados por endocrinólogos.

En el hospital Ramón y Cajal, entonces llamado Centro Especial Ramón y Cajal, Jesús Culebras, en colaboración con el Servicio de Farmacia, cuyo jefe era Domingo García Rodríguez, organizaron una Unidad de Nutricion Parenteral ubicada físicamente en el servicio de Farmacia. Dotada con una campana de flujo laminar, allí se preparaban las mezclas nutrientes que podían servirse a cualquier paciente hospitalizado. El Departamento de Cirugía fue dotado de treinta bombas volumétricas IMED (IMED mod 922 Infusion Pump. Imed Corp, La Jolla, California) y otras tantas peristálticas modelo IVAC. Cuando se requería nutrición parenteral en otros servicios o departamentos se les facilitaban bombas y el correspondiente utillaje. Junto con Domingo García trabajaban las farmacéuticas Milagros Anaya y Maria Ceña quienes desde el primer momento mostraron su especial interés por la nutrición parenteral. Entre la Unidad de Nutricion Parenteral ubicada en el Servicio de Farmacia y la sección de Nutricion liderada por Ana Sastre no había prácticamente relación, simplemente porque en aquella época parecían campos muy distintos y distantes entre sí.

Culebras, en el Departamento de Cirugía, cuyo jefe era Adolfo Núñez Puertas, popularizó la nutrición parenteral entre los pacientes quirúrgicos. En esta dedicación atrajo al grupo a los servicios de Cuidados Intensivos (A. Ascorbe) y de Infecciosas (Emilio Bouza).

En el Hospital Ramón y Cajal se organizaron al menos dos Reuniones de Nutricion Parenteral de Madrid en las que participaron, además de los médicos del hospital Ramón y Cajal, Enrique Rojas, de la Clínica Puerta de Hierro, Cabezas Cerrato del Hospital Clínico de San Carlos y Tomas Caparros de la Fundación Jiménez Diaz (comunicación personal del Dr. Jesus Culebras).

Conscientes de la necesidad imperiosa de desarrollar la nutrición parenteral en los hospitales y a sabiendas de que no había foros científicos adecuados para su difusión y desarrollo, fue en el hospital Ramón y Cajal, bajo la coordinación de Culebras donde se gestó la creación de la Sociedad Española de Nutricion Parenteral y Enteral (SENPE) (2). El día 3 de noviembre de 1977 se dieron de alta los estatutos de SENPE en el entonces existente Ministerio del Interior. Los firmantes que aparecen en el acta fundacional de SENPE son Jesus Culebras Fernández e Isaac Capela Fernández, ambos jefes de Sección del Departamento de Cirugía del Hospital Ramón y Cajal.

Las películas que se muestran a continuación fueron fruto de estas inquietudes y esta colaboración multidisciplinar, como se indica en los fotogramas de créditos.

Las dos películas se mostraron en la IX Semana de Cine Medico de Motril celebrado en la primavera de 1979 en Motril (Granada) obteniendo mención especial y diploma. Se presentaron también en el primer Congreso de la European Society of Parenteral and Enteral Nutrition (ESPEN) celebrado en Estocolmo en $1979(3,4)$ y en la Primera Reunión Nacional de SENPE en Bilbao $(5,6)$.

Existe constancia de que en la Segunda Reunión Nacional de la SENPE, celebrada en Barcelona el 13 y 14 de mayo de 1980 (7) se presentó una película, también en 16 mm, titulada «Catéteres subclavios», cuyos autores eran JM Culebras, P Enríquez, A Badía y M Calvo pero no ha sido posible localizarla. 


\section{EL TRATAMIENTO DE LAS FISTULAS INTESTINALES DE ALTO DEBITO CON NUTRICIÓN PARENTERAL}

Las fistulas intestinales de alto debito son una circunstancia patológica en la que la nutrición parenteral se considera terapéutica primaria y su eficacia está comprobada. La misión de la nutrición parenteral en el tratamiento de las fistulas gastrointestinales a cualquier nivel consiste en promover el cierre de las mismas a base de colocar en reposo el aparato digestivo al tiempo que se suministra un soporte nutritivo adecuado. El uso de nutrición parenteral para estos fines afecta de manera favorable el cierre de las fistulas, con frecuencia sin necesidad de intervención quirúrgica y ha demostrado un efecto muy positivo disminuyendo la mortalidad por esta causa. No es el caso de la fistula que se presenta en la película. Al cabo de más de 100 días de nutrición parenteral y enteral hubo que resecar la fistula y restituir el tránsito intestinal con anastomosis termino terminal.

El tratamiento de las fistulas de alto debito con nutrición parenteral fue brillantemente expuesto y demostrado por Aguirre et al. en 1973 (8).

En 1960, antes de los estudios de Dudrick en el hombre, el niño y en el perro (9), la mortalidad por fistulas oscilaba entre 47 y $62 \%$ (9). El fallecimiento se hallaba directamente relacionado con el flujo de la fístula, la sepsis, la desnutrición y los desequilibrios hidroelectrolíticos. Cuando la desnutrición era grave el exitus del paciente se encontraba prácticamente garantizado.

Con la utilización de la nutrición parenteral la mortalidad por fistula gastrointestinal disminuyó a valores entre 6 y 21 por ciento y el índice de cierre sin intervención quirúrgica se situó por encima del 70 por ciento.

Todavía existe mortalidad en las fístulas intestinales pero con el advenimiento de la nutrición parenteral en el tratamiento de estos pacientes junto con antibioterapia, soporte respiratorio, mejor anestesia y monitorización de los pacientes, control de pH, electrolitos, bicarbonato, etc., la causa principal no es ya la desnutrición sino, habitualmente una sepsis incontrolada.

Las fístulas digestivas a cualquier nivel deben considerarse una indicación princeps de nutrición parenteral. Salvo en los casos que exista obstrucción distal a la fístula, discontinuidad intestinal o abscesos intercurrentes, las posibilidades de que una fístula cierre sin necesidad de cirugía son muy elevadas.

Hay que señalar como indicaciones relativas para la intervención quirúrgica la presencia de enfermedad inflamatoria, la existencia de un drenaje alto persistente, la radiación del área por otro tipo de enfermedades y, finalmente, la epitelización del trayecto fistuloso. Nunca debe soslayarse, en pacientes previamente intervenidos, la posibilidad de presencia de cuerpos extraños que perpetúen la producción de una fístula. Por tanto, estas circunstancias deben investigarse de manera exhaustiva.

Entre los factores que más influyen en el pronóstico y mortalidad en los enfermos con fístulas digestivas tratadas con nutrición parenteral figura la localización anatómica de la misma. Las fístulas yeyunales presentan una mortalidad global del 38 por ciento, frente a una mortalidad de solo 17 por ciento para las fístulas ileales. Las fístulas duodenales ofrecen un cierre espontaneo del 98 por ciento en un tiempo medio de 18,7 días; las fistulas yeyunales cierran espontáneamente en el 87,5 por ciento y las fistulas cólicas en el 69,2 por ciento.

El peor resultado se obtiene en las fistulas ileales que solo cierran espontáneamente en un 40 por ciento. Este bajo porcentaje es atribuible al hecho de que la causa más frecuente de fístula ileal suele ser la ileitis terminal. 
El tratamiento con nutrición artificial de las fístulas gastrointestinales también está sujeto a complicaciones. En la clásica serie descrita a por Aguirre et al de 38 pacientes las complicaciones que se observaron pueden consultarse en la tabla 1.

Tabla 1.- Complicaciones en 38 pacientes portadores de fistula gastrointestinal a diversos niveles según Aguirre et al. (7)

\begin{tabular}{|c|c|c|c|}
\hline Complicación & Gastroduodenal & Intestino delgado & Intestino grueso \\
\hline Sangrado de la fístula & 2 & 2 & \\
\hline Anemia & 1 & 6 & \\
\hline Malnutrición & 3 & 15 & 3 \\
\hline \multicolumn{4}{|c|}{ Complicaciones Pulmonares } \\
\hline Neumonía & 3 & 2 & 1 \\
\hline Embolia pulmonar & & & 1 \\
\hline \multicolumn{4}{|l|}{ Infección } \\
\hline Infeccción de la herida & 3 & 15 & 2 \\
\hline Absceso intraperitoneal & 1 & 9 & 3 \\
\hline Peritonitis & & 1 & 1 \\
\hline Sepsis & 1 & 7 & 2 \\
\hline Desequilibrio electrolítico & 2 & 4 & 1 \\
\hline Obstrucción & & 1 & \\
\hline Dehiscencia de la herida & 1 & 2 & \\
\hline Fracaso renal & & & 1 \\
\hline Ictericia & & & 1 \\
\hline Ulceración de la piel & & 6 & 1 \\
\hline Status depresivo & & 6 & \\
\hline
\end{tabular}




\section{LA NUTRICIÓN PARENTERAL A DOMICILIO (NPD)}

Al contrario de la nutrición enteral domiciliaria (NED), la NPD no está legislada en nuestro país; ello, conjuntamente con el reducido número de centros hospitalarios que disponen de estructura suficiente para abarcar los programas de NPD, contribuye a que la prevalencia de este tratamiento en España se encuentre en cifras muy inferiores a las que se observan en países cultural y económicamente comparables. En 2000, el grupo de trabajo de «Nutrición Artificial, domiciliaria y Ambulatoria (NADYA) de la SENPE se puso como objetivo conseguir un registro de NPD lo más real posible que permitiera un análisis en profundidad de este tratamiento en España. En aquel estudio colaboraron 14 centros hospitalarios recopilando datos de 67 pacientes. La duración media del tratamiento fue de 7,48 $\pm 4,39$ meses. El 59,7\% de los pacientes permanecieron en el programa más de 6 meses, el 19,4\% entre 3 y 6 meses, y en el 20,9\% el tratamiento duró menos de 3 meses. De las complicaciones habidas las más frecuentes fueron las infecciosas, siendo las más destacadas la sepsis por catéter $(0,12 / 100$ días de NPD).

La inclusión en el programa de pacientes neoplásicos incrementaría la serie, aunque teniendo en cuenta la poca prevalencia de la NPD en pacientes cancerosos en 2000 no arrojaría mucha variación (11). En 2014 (12) se registraron 220 pacientes, con 229 episodios de NPD, procedentes de 37 hospitales, lo que representa una tasa de 4,73 pacientes/millón de habitantes/ año 2014. Las patologías más frecuentes en los adultos fueron: "otros diagnósticos" (22,3\%), neoplasia paliativa $(20,4 \%)$, neoplasia activa radical $(11,8 \%)$ e isquemia mesentérica $(10,9 \%)$. En los niños las patologías más frecuentes fueron: "alteraciones congénitas intestinales" (33,3\%), seguidas por intestino corto traumático y "otro diagnóstico". Los autores consideraron, como ya había hecho el grupo NADYA en 2004 y como se sugiere en la película en 1978, que la NPD debería ser regulada por la Administración Sanitaria en el marco del Consejo Interterritorial del Sistema Nacional de Salud y que su inclusión en la Cartera de Servicios de los diferentes Servicios de Salud de las diferentes comunidades autónomas redundaría en un beneficio para el paciente y los profesionales.

\section{DESCRIPCIÓN DE LAS PELÍCULAS}

\section{Película 1: Nutricion parenteral y enteral en un fistula yeyunal alta}

Dirección de acceso a la película:

https://drive.google.com/file/d/1D7GYTVJqerFmbNdpzCpNyLHdPAo_OHF2/view?usp=sharing

Dirección abreviada de acceso a la película:

https://goo.gl/1DQ2zE

Ficha técnica:

Película en 16 mm, color, duración 15 minutos.

Cámara: E. Llosa. Cine científico. Rio 6, Madrid

Departamento de Cirugía General y Digestiva (Jefe: A Núñez Puertas). Servicio de Farmacia (Jefe: Domingo Garcia Rodriguez) Servicio de Cuidados Intensivos quirúrgicos (Jefe: A. Ascorbe). Sección de Enfermedades Infecciosas (Jefe: E Bouza) 
Autores: Jesus M. Culebras, Virgilio Fresneda, Pablo Enríquez Valens, C Botella, A Buzón, Maria Ceña, José Nistal, Alfredo Badia, JM Maudes, Milagros Anaya, Blanca Megia

Sinopsis:

La película se inicia mostrando una paciente de 32 años que lleva dos meses con nutrición parenteral exclusiva después de dos intervenciones a raíz de un aborto séptico con perforación de útero y de un asa intestinal. En la segunda intervención se le practicó histerectomía. En el postoperatorio hizo una dehiscencia de pared y fístula yeyunal alta que drenaba por vagina y por varios trayectos fistulosos a la pared abdominal. Por las fístulas llegó a salir $1800 \mathrm{ml}$ al día. Hizo también varios episodios de sepsis con diversas cepas de gérmenes rebeldes...

Pero, curiosamente, los autores cambian el plano porque no es de esta paciente de la que van a hablar sino de la mujer que la visita, Fuencisla, de 36 años quien un año antes pasó por un trance similar. Casada y embarazada de cuatro meses, presentó un cuadro de apendicitis aguda. Un absceso peritoneal postoperatorio le produjo una oclusión por lo que fue laparotomizada. El cierre de la pared se vio dificultado por el embarazo. Al cuarto día presentó un cuadro séptico con fiebre alta y gérmenes aerobios y anaerobios. Abortó espontáneamente. Presentó un gran absceso de pared que se desbridó y una insuficiencia respiratoria severa que precisó intubación con presión positiva. Como consecuencia hubo una eventración con salida de asas intestinales y una fistula yeyunal alta de elevado flujo que llegó a alcanzar $2575 \mathrm{ml}$ en 24 horas. Para controlarla estaba sometida a monitorización cardiaca, presión venosa central, control horario de diuresis y colchón hidráulico antiescara.

La enferma engordó aun a pesar de su situación séptica. Su peso corporal se monitorizaba diariamente con una balanza de precisión. La herida, que en un principio medía $11 \mathrm{~cm}$ de borde a borde, fue mejorando paulatinamente. La pared fue protegida con pasta Lassar y se irrigaba con solución de Tremoliere. Aspiración nasogástrica continua. En previsión de una larga enfermedad y a pesar de tener un estado nutritivo muy bueno, se le administraban por vía parenteral 4000 calorías y $10 \mathrm{~g}$ de nitrógeno al día por término medio. Recibía además grandes cantidades de albúmina y de sangre. Aspiración continua. La paciente se hace hemodinámicamente estable y por ello es trasladada a planta de cirugía dónde continuará con los cuidados. Sigue manteniéndose la aspiración continua sobre la herida abdominal con una sonda de Pezzer.

Pero los problemas se suceden uno detrás de otro por lo que se vieron obligados a prescindir de la vía venosa central. Se decidió ensayar una alimentación enteral continua a través de su fístula con la ayuda de una sonda lastrada de Dobhoff durante 53 días que resultó satisfactoria lo que permitió que la herida se redujera exclusivamente a la boca fistulosa y por tanto en condiciones idóneas para una intervención quirúrgica correctora. Los procesos infecciosos múltiples que presentó la enferma hicieron sospechar un déficit del sistema inmuno competente. Sin embargo, el estudio de la función de los linfocitos T, la transformación blástica con fitohemaglutininas, la formación de rosetas, un test cutáneo con PPD etc., pusieron de manifiesto una función inmunológica correcta.

Se tuvo en cuenta la rehabilitación física. En la última parte de su hospitalización la enferma deambulaba por los alrededores del hospital desconectándose temporalmente de la nutrición artificial.

Finalmente, se decidió la intervención: laparotomía media suprainfraumbilical sobre anterior incisión. Liberación de adherencias múltiples y resección de $15 \mathrm{~cm}$ de intestino. Reconstrucción de transito con anastomosis termino terminal. Biopsia hepática en la que la anatomía patológica confirmó áreas de esteatosis zonal. Cierre de la pared con puntos totales

Como resumen del proceso diremos que a lo largo de 130 días de hospitalización la paciente presentó una apendicitis aguda gangrenada, un absceso intraperitoneal, un absceso de pared, una infección de pared, infección postquirúrgica mixta aerobia y anaerobia, sepsis por enterococo 
y varios hemocultivos positivos tratados con cloranfenicol y gentamicina, sepsis por Acinetobacter calcoaceticus en relación con catéter que no precisó antibioterapia, una sepsis estafilocócica con estafilococo coagulasa positivo, también en relación con catéteres, una endocarditis derecha, embolismos sépticos pulmonares repetidos, un nódulo pulmonar de naturaleza infecciosa, hígado de sepsis con bilirrubina alta y fosfatasa alcalina alta, transaminasas normales, defectos de captación en la gammagrafia, dos abscesos glúteos, otro absceso de pared, 43 días con fiebre de más de 38 ${ }^{\circ} \mathrm{C}$ a pesar del tratamiento con antibióticos y monitorización en sangre de la concentración de los mismos. No obstante todos estos incidentes la enferma recuperó el tránsito intestinal y mantuvo el peso entre 57 y $58 \mathrm{~kg}$.

Dos meses después del alta tuvo una regla menstrual normal, otra prueba más de su restitutio ad integrum.

La nutrición parenteral y enteral fue crucial en la evolución. La fístula residual hubo de resolverse quirúrgicamente. Un año después la paciente se encontraba perfectamente. Únicamente presentaba una cicatriz abdominal totalmente cerrada que no reflejaba ni mucho menos las vicisitudes pasadas.

\title{
Película 2: Intestino artificial (nutrición parenteral ambulante)
}

\author{
Dirección de acceso a la película: \\ https://drive.google.com/file/d/1mPkXunM1N_Saboor-EdNMelnidXEINHy/view?usp=sharing \\ Dirección abreviada de acceso a la película:
} https://goo.gl/X2nXyp

\section{Ficha técnica:}

Película en 16 mm, color, duración 10 minutos y 38 segundos.

Cámara: E. Llosa. Cine científico. Rio 6, Madrid

Departamento de Cirugía General y Digestiva (Jefe: A Núñez Puertas). Servicio de Farmacia (Jefe: Domingo Garcia Rodriguez) Servicio de Cuidados Intensivos quirúrgicos (Jefe: A. Ascorbe)

Autores: Jesus M Culebras, Virgilio Fresneda, María Ceña, María Calvo, Milagros Anaya. Pablo Enriquez Valens, Blanca Mejia.

\section{Sinopsis:}

La pelicula, filmada en 1978, empieza con unas vistas de la plaza de toros Monumental de las Ventas, de Madrid y aparecen tres personas trajeadas charlando. Una voz en off dice que uno de ellos es portador de un cateter permanente para someterse a nutrición parenteral como metodo exclusivo de alimentacion. Las imágenes que siguen muestran varias secuencias radiológicas de la lesion cancerosa del paciente. A continuacion aparece una secuencia retrospectiva que enseña cómo se colocó el caterer central a traves de la vena cefálica, una rama venosa de la vena subclavia derecha. Un cateter fabricado de silicona se introduce por la parte proximal de la vena cefálica mientras que el otro extremo se tuneliza por la region subcutanea antepectoral hasta exteriorizarse a la altura y lateralmente al pezón. De este modo se consiguen dos cosas: disminuir el riesgo de contaminacion venosa por el caterter y facilitar al paciente el acceso al cateter. Este modelo de cateter es el que utilizaban Solassol y Joyeux en Montpellier, Francia (13).

Las imágenes que siguen muestran la Unidad de Nutrición Parenteral del hospital Ramon y Cajal. Las unidades nutrientes son facilitadas al paciente. En el domicilio, un familiar instruido en 
la Unidad de Nutricion Parenteral custodia la solución nutriente. En una de las secuencias puede verse la bolsa de nutrición con la fecha de caducidad, que en este caso es 27/10/78 (ver figura 1).

Figura 1. Detalle de la unidad parenteral nutriente de 3 litros: Indica la composición y fecha de caducidad, en este caso 27/10/78

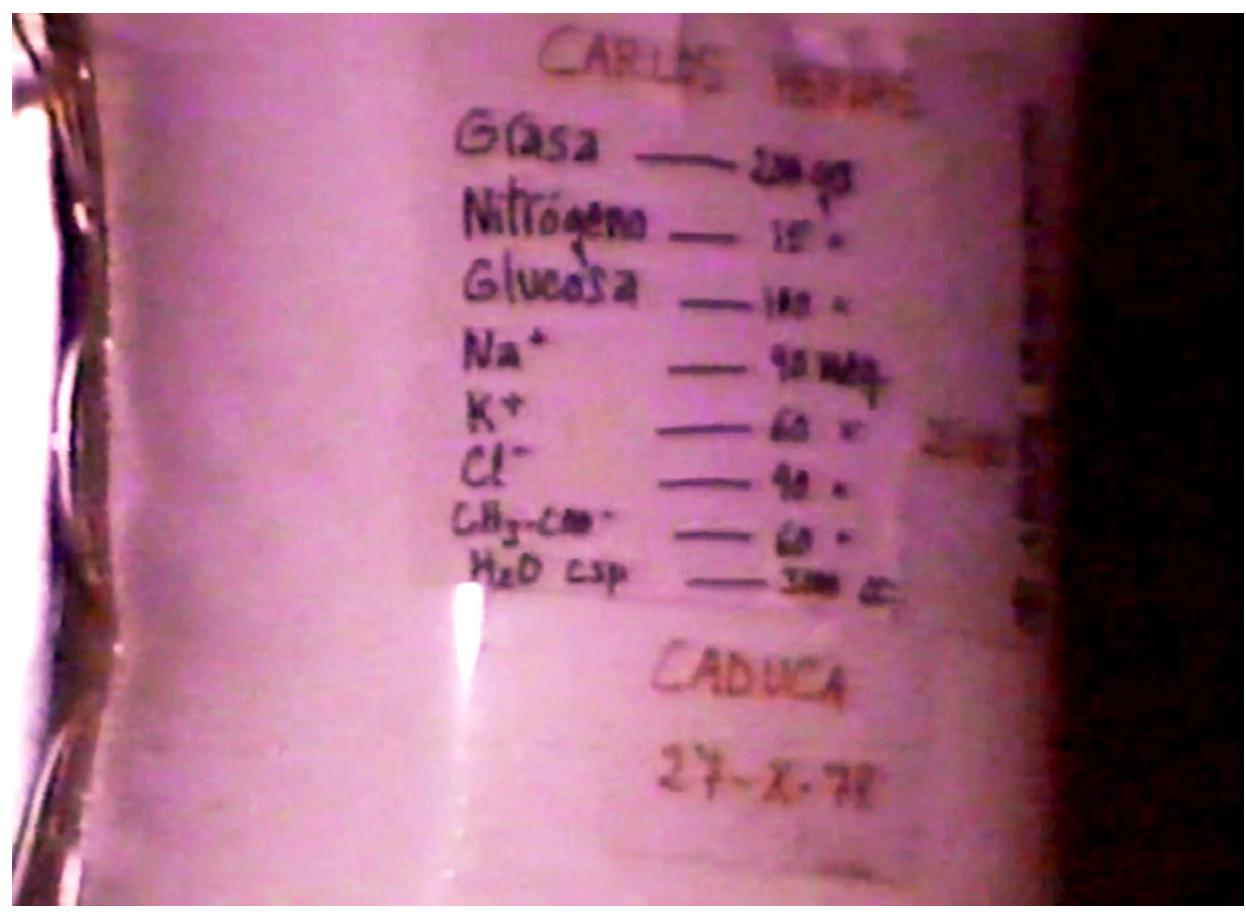

Finaliza la pelicula mostrando los detalles de todas las conexiones y la voz en off hace el siguiente comentario:

El modelo de intestino artificial esta dispuesto para su utilizacion en pacientes como éste y sobre todo para aquellos otros que tengan el tracto digestivo definitivamente anulado por enfermedad no tumoral y por tanto susceptibles en teoria de supervivencia indefinida. La nutricion parenteral es una tecnica cara pero necesaria. La nutricion parenteral ambulatoria supone un enorme ahorro en días de hospitalizacion y por ello debe ser potenciada. Pero, no debemos contentarnos con tener un modelo. Es preciso organizar de inmediato una infraestructura asistencial domiciliaria especializada y determinar el sistema de financiacion de la nutricion parenteral ambulante.

Esta película ha pasado desapercibida en la historia de la nutricion artificial en España. Prueba de ello es que en un articulo de Moreno Villares (14) se afirmaba:

La NPD se utilizó por primera vez en 1967 en Estados Unidos de Norteamérica. Los primeros pacientes europeos datan de principios de los años 1970 en Dinamarca, mientras que hay que esperar hasta 1984 para encontrar los primeros casos en España. 
Como se desprende de lo reseñado en este articulo, a finales de la década de los años setenta del siglo XX había en España varios equipos dedicados a la nutricion artificial, fundamentalmente compuestos por cirujanos, con un nivel de interés y dedicación a la nutrición artificial similar al de otros paises de Europa y America.

\section{BIBLIOGRAFÍA}

1. Vara Thorbeck C, García Aranguez L. Nutrición completa por vía parenteral: principios básicos de hiperalimentación. Madrid, España: Editorial Marban; 1976.

2. Jesús Culebras. Treinta y seis años al frente de una revista científica: Memorias del director de Nutrición Hospitalaria. León, España: Universidad de León, Area de Publicaciones; 2017.

3. Culebras JM. Scientific Cinema: Parenteral and Enteral Nutrition in a high output jejunal fistula. In: First European Congress on Parenteral and Enteral Nutrition. Stockholm, Sweden: European Society for Clinical Nutrition and Metabolism (ESPEN); 2-5 September 1979.

4. Culebras JM. Scientific Cinema: Artificial Gut (Home parenteral nutrition). In: First European Congress on Parenteral and Enteral Nutrition. Stockholm, Sweden: Europena Society for Clinical Nutrition and Metabolism (ESPEN); 2-5 September 1979.

5. Culebras JM. Sesión de cine científico: Nutricion parenteral y enteral en una fistula yeyunal alta. En: Primera Reunión Nacional de la Sociedad Española de Nutricion Parenteral y Enteral (SENPE). Bilbao, España: SENPE; 25 y 26 de mayo de 1979.

6. Culebras JM. Sesión de cine científico: Intestino artificial ( Nutricion Parenteral ambulante). En: Primera Reunión Nacional de la Sociedad Española de Nutricion Parenteral y Enteral (SENPE). Bilbao, España: SENPE; 25 y 26 de mayo de 1979.

7. Segunda Reunión Nacional de la Sociedad Española de Nutricion Parenteral y Enteral (SENPE). Barcelona, España: SENPE; 13 y 14 de mayo de 1980.

8. Aguirre A, Fischer JE, Welch CE. The role of surgery and hyperalimentation in therapy of gastrointestinal-cutaneous fistulae. Ann Surg. 1974;180(4):393-401. PMID: 4213479

9. Dudrick SJ, Wilmore DW, Vars HM, Rhoads JE. Long-term total parenteral nutrition with growth, development, and positive nitrogen balance. Surgery. 1968;64(1):134-42. PMID: 4968812

10. Edmunds LH Jr, Williams GM, Welch CE. External fistulas arising from the gastro-intestinal tract. Ann Surg. 1960;152(3):445-71. PMID: 13725742

11. Planas M, Castellá M, León M, Pita A M, García Peris P, Gómez Enterría P et al; Grupo NADYA-SENPE. Nutrición parenteral domiciliaria (NPD): registro NADYA, año 2000. Nutr Hosp. 2003; 18(1):29-33.

12. Wanden-Berghe C, Pereira Cunill JL, Cuerda Compes C, Moreno Villares JM, Pérez de la Cruz A, Burgos Peláez R, et al; Grupo NADYA-SENPE. Nutrición parenteral domiciliaria en España durante 2014: informe del Grupo de Nutrición Artificial Domiciliaria y Ambulatoria NADYA. Nutr Hosp. 2015;32(6):2380-4. DOI: 10.3305/nh.2015.32.6.10056; PMID: 26667683

13. Solassol C, Joyeux H. El intestino artificial en el cáncer gastrointestinal. Boletín de la SENPE. 1980;1(2):129-42.

14. Moreno Villares JM. La práctica de la nutrición artificial domiciliaria en Europa. Nutr Hosp. 2004;19(2):59-67. PMID: 15049406 